\title{
Chikungunya virus in Canada
}

\section{A case report highlighting the need for increased global health education}

\author{
Herman Bami, Jason L Elzinga \\ Faculty Reviewer: Javeed Sukhera, HBSc, MD, DABPN, FRCSC (Department of Psychiatry)
}

\begin{abstract}
This article presents a previously reported case involving the first Canadian patient to acquire Chikungunya virus (CHIKV) infection after travelling to a newly endemic region in the Americas. The specific history and clinical presentation of this patient is examined, including the treatment and complete resolution of the patient's symptoms. A brief overview of the general disease course and diagnosis of CHIKV is provided. This case emphasizes the importance of global health education in Canadian medical curricula. The current standards of global health education in Canadian medical schools are briefly reviewed and recommendations based on expert opinions are provided. Although such programs exist, their implementation was found to be variable between schools and increased attention and standardization is currently required.
\end{abstract}

\section{CASE PRESENTATION}

A 57-year-old Caucasian male with no previous medical history travelled to Martinique, an island in the East Caribbean, from mid-January to early February 2014. ${ }^{1}$ Around 3 days after his return to Quebec, he presented with several symptoms including fever, myalgia, and increasingly progressive headaches. ${ }^{1}$ The patient sought medical attention with his primary complaints being arthralgia in his extremities, joint swelling and a nonpetechial rash on his thorax. ${ }^{1}$ Upon further questioning, the patient reported that while in Martinique, he resided in a villa close to the mountains. ${ }^{1}$ Although the villa itself was reportedly well maintained, the patient reported mosquito bites, mostly during the first few days of his approximately 2 -week stay. ${ }^{1}$ The patient did not experience episodes of fever, gastrointestinal or respiratory illness during his trip. ${ }^{1} \mathrm{He}$ was evaluated for renal and liver anomalies with no significant results. ${ }^{1}$

Serological testing for dengue virus was ordered on paired sera samples with negative results for the anti-dengue IgM and IgG antibodies. ${ }^{1}$ The patient was treated supportively with nonsteroidal anti-inflammatory drugs. ${ }^{1}$ Upon treatment, the fever subsided, however musculoskeletal symptoms persisted for an additional 2 weeks. ${ }^{1}$

Four weeks after the initial symptoms, the patient received follow-up blood tests. ${ }^{1}$ The patient reported gradual improvement in symptoms, although arthralgia and residual morning joint stiffness were both still present. ${ }^{1}$ Physical examination displayed no signs of synovitis and a presumptive diagnosis of arbovirus infection was given. ${ }^{1}$ Additional serological testing for Rickettsia spp. and chikungunya virus (CHIKV) was conducted with a positive CHIKV IgM enzyme-linked immunoassay found. ${ }^{1,2}$ The positive finding was lat- er confirmed by a CHIKV plaque neutralization test. ${ }^{1}$ Amplification of the CHIKV envelope EI gene from the samples was initially attempted by reverse-transcriptase polymerase chain reaction. ${ }^{1,3}$ Successful amplification of the targeted genome resulted in a product that could be sequenced. ${ }^{1}$ These results were consistent with initial findings related to an Asian strain circulating in the Caribbean.

Eight weeks following his first patient visit, the patient reported returning to his previous state of health with a lack of residual febrile syndrome, and absence of both hand stiffness and other symptoms. ${ }^{1}$

\section{DESCRIPTION OF CHIKV: PATHOGENESIS AND EPIDEMIOLOGY}

Chikungunya is an RNA alpha virus of the Togaviridae family that is transmitted by the Aedes aegypti and Aedes albopictus mosquitos. ${ }^{4}$ Although the virus is endemic in nonhuman primates, humans can also serve as primary hosts during periods of acute illness that allow for infection of biting mosquitos. ${ }^{5}$ The virus was first isolated in Tanzania in 1953 but spread to Thailand in 1958. ${ }^{6} \mathrm{In}$ 2004, an outbreak sparked a transmission to Mozambique, India, Sri Lanka and other parts of Southeast Asia. ${ }^{7}$ The virus first reached the Western hemisphere in late 2013 with transmission to the Caribbean island of St. Martin. ${ }^{6}$ Since then, it has reached up to 44 countries including the United States and Canada., ${ }^{1,8}$

CHIKV infection is characterized by an acute onset of high fever in addition to symmetric and severe polyarthralgia in the small distal joints. ${ }^{4}$ Other possible symptoms include headache, myalgia, and vomiting with further examination potentially revealing arthritis, lymphadenopathy, and conjunctivitis. ${ }^{5,9}$ The most common laboratory finding is lymphopenia, the severity of which correlates with the extent of viremia. ${ }^{9} \mathrm{CHIKV}$ has an incubation period of 2 to 12 days with the subsequent viremia lasting between 4 to 7 days. ${ }^{4} \mathrm{Al}-$ though acute symptoms typically do not last more than 2 weeks, up to $60 \%$ of patients can experience relapsing and severe arthralgias for months to years after the infection subsides. ${ }^{5,7}$ While CHIKV has a low mortality rate, elderly and immunocompromised patients are at a higher risk for life-threatening disease including meningoencephalitis, hepatitis, myocarditis and nephritis. ${ }^{5}$ Differential diagnoses often include dengue virus, which carries similar symptoms (see Figure 1) and is transmitted by the Aedes mosquito in similar locations. ${ }^{10}$ Additionally, chronic arthralgia may mimic rheumatologic conditions including rheumatoid arthritis, underscoring the need a comprehensive history upon initial presentation. ${ }^{10}$

Although malaria is still the most common cause of fever in travellers returning to Canada, a broader spectrum of other vector-borne and viral infections are being detected over time including measles, CHIKV and dengue virus. ${ }^{11}$ Since early 2014 and the 
initial transmission of the CHIKV to the Caribbean islands, a dramatic increase in CHIKV cases diagnosed in Canada has been seen, emphasizing the need for increased awareness and education for Canadian clinicians and healthcare providers. ${ }^{12}$

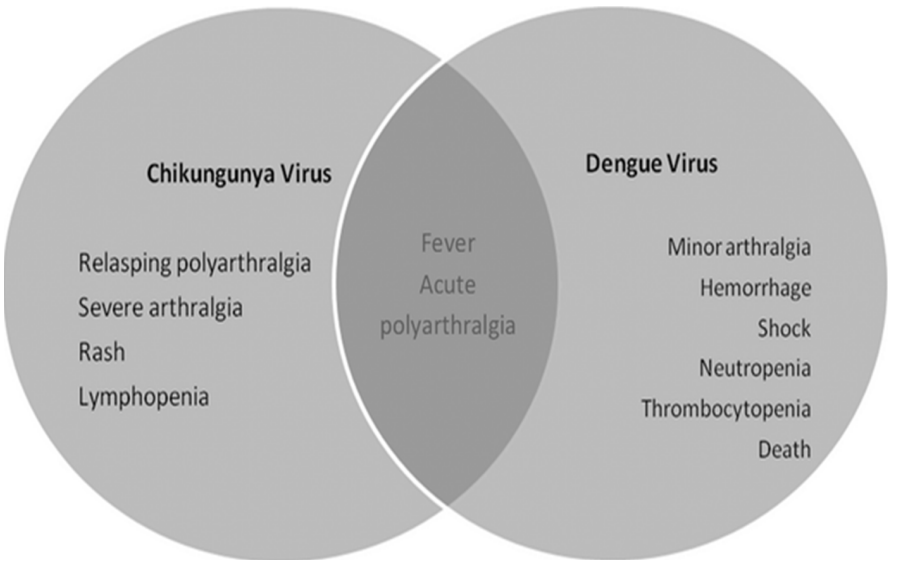

Figure 1. Clinical presentations of CHIKV and dengue virus including overlapping and distinguishing clinical signs. ${ }^{10}$

\section{GLOBAL HEALTH EDUCATION}

\section{Need for increased training}

Due to trends in globalization and travel bringing previously foreign pathogens to North America, students and medical educators should maintain a global perspective on infectious illness needs to mitigate the potentially negative societal impact of globalization. Globalization is a multifaceted phenomenon with a myriad of potential and critical health impacts, ranging from direct impacts on individuals through healthcare delivery to indirect impacts via economic and other social factors. ${ }^{13}$ For instance, globalization resulted in liberalization of the airline industry, leading to a dramatic increase in global air travel, thus enabling the rapid spread of communicable disease. ${ }^{13}$ In the past 5 years, numerous emerging infective agents such as Zika virus, Ebola virus, Enterovirus D68, and CHIKV have spread broadly and rapidly. These infections emphasize the need for increased training and awareness of global health issues. Furthermore, the rapid influx of newcomers due to international conflict, especially considering the recent crisis in Syria, highlights the importance of such training.

While "global health" can be an evasive term, especially when used with terminology such as "international health" or "tropical medicine", it is increasingly being used to denote the health issues that transcend national borders, race, ethnicity, income or culture. ${ }^{14}$ Although significant differences exist between disease patterns based on geography, the factors that perpetuate disease states which include poverty, political instability, limited access to care and genetic determinants - are often quite related. ${ }^{14}$ This similarity can perhaps be best exhibited by the rise of chronic conditions such as cardiovascular and lung disease in developing countries due to increased risk of smoking, poverty and educational factors..$^{14}$ In order for medical students to be able to address these complex and nuanced issues, there has to be a clear and comprehensive integration of global health in medical school curricula.

\section{Current status and recommendation}

The past few years have brought a substantial increase in medical students interested in global health. ${ }^{14,15}$ In a recent study conducted on American medical schools, it was found that many matriculating students had prior international experiences, and up to $30 \%$ chose to partake in international electives during their studies. ${ }^{15}$ Additionally, up to $68 \%$ of schools had active student global or international health groups. ${ }^{15}$ In a similar study in Canada, 53\% of the medical schools had either specific global health lectures or modules as part of the mandatory courses. ${ }^{16}$ As a caveat, there is significant variation across schools in the material covered, the amount of information provided, and the year in which this training is offered. ${ }^{16}$ While all schools offer the opportunity to take part in international electives, there has historically been poor consistency in the pre-departure training provided, as well as the financial and organizational support provided by the school. ${ }^{16}$ Despite the growing interest exhibited by medical students and the increased need for global health training, medical school curricula have yet to deliver a sufficient and coherent response. ${ }^{15,16}$

Recommendations have been made for the foundations of global health curricula in undergraduate medical programs. In a 2010 update to guidelines released by the Association of Faculties of Medicine of Canada Resource Group on Global Health/GHEC joint committee, the group proposed all medical graduate should have competency in the following areas: global burden of disease, health implications of travel, social and economic determinants of health, population health, globalization of healthcare, healthcare in low-resource settings, and human rights in global health. ${ }^{15}$ Another study based on work from the American Society for Tropical Medicine and Hygiene Committee on Medical Education consolidated the core competencies of global health education into 3 domains: burden of global diseases, traveller's medicine, and immigrant health. ${ }^{15}$ Despite these consensus competencies, tremendous variability remains.

Despite the difficulty of incorporating novel elements into medical school curricula, and ensuring that these newer offerings are available in learning formats accessible to all students, it is critical to ensure that physicians graduating today have the skills to make them competent and effective practitioners. While standardized curricula of a specific length or format may be impractical for all medical schools to accommodate, increased student interest in global health and the increased demands on today's medical practitioners require action.

\section{CONCLUSION}

This report presents the first case of a Canadian acquiring CHIKV after travelling to a newly endemic region in the Western hemisphere. The clinical picture of this disease is also examined. While other potential causative infectious agents can confound diagnoses, it is important for clinicians to be aware of the different presentations of emerging infections. In addition, this case highlights the continual need for global health education in its varied formats for medical trainees. There is ongoing debate regarding 
standards for global health education in Canadian medical schools, with a number of recommendations being put forward from expert opinion groups.

\section{REFERENCES}

1. Therrien C, Jourdan G, Holloway K, et al. First Imported Case of Chikungunya Virus Infection in a Travelling Canadian Returning from the Caribbean. Can J Infect Dis. 2016 Feb 24;2016.

2. Martin DA, Muth DA, Brown T, et al. Standardization of immunoglobulin $\mathrm{M}$ capture enzyme-linked immunosorbent assays for routine diagnosis of arboviral infections. J Clin Microbiol. 2000 May 1;38(5):1823-6.

3. Machine TP. Movement of Asian Genotype Chikungunya Virus. DNA. 2003;41:3494-8.

4. Hamer DH, Chen LH. Chikungunya: Establishing a New Home in the Western Hemisphere Chikungunya. Ann Intern Med. 2014 Dec 2;161(11):827-8.

5. Staples JE, Fischer M. Chikungunya virus in the Americaswhat a vectorborne pathogen can do. New Engl J Med. 2014 Sep 4;371(10):887-9.

6. Morrison TE. Reemergence of chikungunya virus. J Virol. 2014 Oct 15;88(20):11644-7.

7. Stamm LV. Chikungunya: emerging threat to the United States. JAMA Derm. 2015 Mar 1;151(3):257-8.

8. Geographic Distribution [Internet]. Centers for Disease Control and Prevention; 2016 April 22 [cited 2017 Mar 29]. Available from: https:// www.cdc.gov/chikungunya/geo/index.html.

9. Weaver SC, Lecuit M. Chikungunya virus and the global spread of a mosquito-borne disease. New Engl J Med. 2015 Mar 26;372(13):1231-9.

10. Peper SM, Monson BJ, Van Schooneveld T, et al. That Which Bends Up: A Case Report and Literature Review of Chikungunya Virus. J Gen Intern Med. 2016 May 1;31(5):576-81.

11. Kariyawasam R, Lau R, Eshaghi A, et al. Spectrum of viral pathogens in blood of malaria-free ill travelers returning to Canada. Emerg Infect Dis. 2016 May;22(5):854.

12. Drebot MA, Holloway K, Zheng H, et al. Travel-related chikungunya cases in Canada, 2014. Can Communic Dis Rep. 2015 Jan 8;41(1):2.

13. Pang T, Guindon GE. Globalization and risks to health. EMBO Rep. 2004 Oct 1;5(1S):S11-6.

14. Houpt ER, Pearson RD, Hall TL. Three domains of competency in global health education: recommendations for all medical students. Acad Med. 2007 Mar 1;82(3):222-5.

15. Khan OA, Guerrant R, Sanders J, et al. Global health education in US medical schools. BMC Med Educ. 2013 Jan 18;13(1):3.

16. Izadnegahdar R, Correia S, Ohata B, et al. Global health in Canadian medical education: current practices and opportunities. Acad Med. 2008 Feb 1;83(2):192-8. 